\title{
LES NOUVELLES FORMES D'ORGANISATION (NFO) : DES SYSTÈMES HOMÉOSTASIQUES DE RÉSILIENCE STRATÉGIQUE
}

\author{
Author(s) / Auteur(s) : \\ Alphonse-Christian IVINZA LEPAPA \\ Docteur en Sciences de Gestion (ULB, Solvay, 2007) \\ DES en Télématique et Organisation (ULB, Faculté des sciences, 1997) \\ Professeur Honoraire de Gestion de la FWB (Belgique). \\ Professeur de Télématique et des Systèmes d'Information de Gestion (MIS) de l'ESU de la RDC. \\ ivinza@hotmail.com
}

\begin{abstract}
Résumé :
Les Nouvelles formes d'organisation (NFO) proviennent d'une nécessité régulière de garder la performance dans un environnement changeant. L'environnement actuel qui est dominé par les technologies de l'information et des télécommunications (TIC) a engendré la Net-économie. Désormais, les entreprises s'organisent autrement : il s'agit de la transition des formes d'entreprises pour assurer leur survie. Déjà Mintzberg (1979) introduisait la notion de transition dans les configurations structurelles.

Les entreprises et les organisations sont considérées comme un système en interaction avec son environnement. Seguin et Chanlat (1983, p. 26) définissent «l'organisation comme un système qui importe certains éléments de son environnement, les transforme et les exporte vers l'environnement».

Le changement organisationnel ou la transition structurelle dont il est question n'est pas naturel dans le sens où il ne s'agit pas d'un acte de gestion courante et opérationnelle. Mais, il s'effectue sous la pression de l'environnement, qui est une contrainte, c'est-à-dire un acte de gestion stratégique. Face aux transformations découlant de cette contrainte, il devient impérieux de redéfinir une nouvelle structure organisationnelle à adopter pour assurer la survie de l'entreprise (transition temporelle diachronique). La transition structurelle trouve son explication dans plusieurs éléments : le rapport avec l'environnement, alors le système est ouvert. La conservation des systèmes, assurer la survie.

Les entreprises sont des systèmes ouverts d'apprentissage à décideurs multiples qui doivent assurer leur conservation et leur survie. Deux concepts permettent d'illustrer la survie des organisations : l'état stationnaire mieux utilisé en cybernétique et l'homéostasie découverte par le médecin français Claude Bernard. Aussi, nous approcherons le changement organisationnel comme un phénomène homéostasique.

L'homéostasie est un processus complexe et autonome de régulation et d'équilibration dans les systèmes vivants. C'est "l'ensemble des processus organiques qui agissent pour maintenir l'état stationnaire de l'organisme, dans sa morphologie et dans ses conditions intérieures, en dépit des perturbations extérieures.» (Durand, 2006, p.19).

L'entreprise est un organisme vivant qui réagit aux changements et cherche à en tirer parti pour s'assurer un avantage sur ses concurrents. Deux facteurs exogènes poussent la création des nouvelles formes d'organisation (NFO) : les TIC et la Nouvelle Economie.

L'idée d'un impact organisationnel des TIC semble relever d'un déterminisme technologique renaissant de ses cendres comme à chaque grande vague d'innovations technologiques. (Kéfi et Kalika, 2005, p. 45). Ayant acquis déjà les économies d'échelle, les NFO recherchent aujourd'hui l'économie d'adaptabilité.

Le concept de résilience provient des travaux de recherche du docteur Borris Cyrulnik sur la capacité et le potentiel des enfants. En psychologie comme en management, la résilience représente la capacité et les attitudes d'un individu ou d'un système à surmonter l'incertitude.

En management on parlera de résilience stratégique qui n'est pas une manière de répondre à une crise passée. Pour Garry Harmel et Liisa Valikangas, elle concerne la capacité d'anticiper le changement avant que la nécessité de changer devienne une évidence. Dans cet article, nous présenterons quelques modèles homéostasiques utilisant les TIC pour la résilience stratégique pour assurer la survie des organisations par une transition structurelle (diachronique).
\end{abstract}

\section{Keywords / Mots-clés :}

nouvelle forme d'organisation (NFO), homéostasie, système homéostasique, résilience, résilience stratégique, TIC, changement organisationnel, transition structurelle 
Dans cet article, nous présentons d'abord dans la première section les Nouvelles Formes d'Organisation (NFO) et les entreprises suivant une conception systémique. Les origines et les catalyseurs des NFO sont abordées.

La seconde section «Homéostasie dans les organisations» présente les concepts suivants: le changement organisationnel, la transition structurelle, l'état stationnaire et l'homéostasie.

La troisième section aborde le concept de résilience stratégique.

La quatrième section présente quelques modèles homéostasiques utilisant les TIC pour la résilience stratégique (MIT90, Triangle stratégique, Holy grail of IT, SAM-Strategic Alignment Model) pour assurer la survie des organisations par une transition structurelle (diachronique).

\section{LES NFO, COMME ENTREPRISES SYSTÈMES}

Cette section présente succinctement les origines et les catalyseurs des NFO et rappelle aussi notre conception des entreprises et des organisations du point de vue systémique.

\section{Origine des NFO}

Les Nouvelles formes d'organisation (NFO) proviennent d'une nécessité régulière de garder la performance dans un environnement changeant. L'environnement actuel qui est dominé par les technologies de l'information et des télécommunications (TIC) qui a engendré la Net-économie ou l'économie numérique.

Désormais, les entreprises s'organisent autrement : entreprise numérique, entreprise réseau, entreprise virtuelle, télétravail, etc. Il s'agit de la transition des formes d'entreprises pour assurer leur survie.

\section{Les entreprises et les organisations comme système}

Les entreprises et les organisations sont considérées, selon l'école systémique du paradigme fonctionnaliste, comme un système en interaction avec son environnement. Seguin et Chanlat (1983) définissent «l'organisation comme un système qui importe certains éléments de son environnement, les transforme et les exporte vers l'environnement».

D'autre part, Luc Wilkin (1999-2000, p.180) considère que «les organisations sont des systèmes ouverts, dont le mode de fonctionnement interne doit être adapté aux caractéristiques de l'environnement». Pour qu'une organisation soit en interaction avec son environnement, il faut qu'elle soit considérée comme un système ouvert, celui pour lequel la survie dépend de l'extérieur.

\section{Les catalyseurs des NFO}

L'entreprise est un organisme vivant qui réagit aux changements et cherche à en tirer parti pour s'assurer un avantage sur ses concurrents. Deux facteurs exogènes poussent la création des nouvelles formes d'organisation (NFO) :

- les TIC (réseaux informatiques, connectivité, interopérabilité, haut débit) ;

- la Nouvelle Economie (recherche de la compétitivité et des performances ; globalisation des marchés des produits et des ressources).

L'idée d'un impact organisationnel des semble relever d'un déterminisme technologique renaissant de ses cendres comme à chaque grande vague d'innovations technologiques. (Kéfi et Kalika, 2005, p.45). Ayant acquis déjà les économies d'échelle, les NFO recherchent aujourd'hui l'économie d'adaptabilité. Ces entreprises préfèrent investir en système d'information que d'acheter un mobilier de luxe.

\section{HOMÉOSTASIE DANS LES ENTREPRISES ET ORGANISATIONS}

Pour la bonne compréhension de notre approche, nous apportons quelques éclaircissements sur les expressions changement organisationnel et transition structurelle. A la fin de cette section, nous contrastons les concepts état stationnaire et l'homéostasie du point de vue organisationnel. 


\title{
Changement organisationnel ou transition structurelle
}

\author{
$1^{\circ}$ Le changement organisationnel
}

Le changement organisationnel ou la transition structurelle dont il est question n'est pas naturel dans le sens où il ne s'agit pas d'un acte de gestion courante et opérationnelle. Mais, il s'effectue sous la pression de l'environnement, qui est une contrainte, c'est-à-dire un acte de gestion stratégique. Face aux transformations découlant de cette contrainte, il devient impérieux de redéfinir une nouvelle structure organisationnelle à adopter pour assurer la survie de l'entreprise (transition temporelle diachronique).

La crise provoquée par cette contrainte peut être «conçue comme une rupture créatrice. C'est un facteur de propagation des méthodes modernes. Le changement organisationnel est une condition pour que le changement stratégique réussisse.» (Godelier, 1998, p.26 et sq.)

Le changement organisationnel ou la transition structurelle trouve son explication dans plusieurs éléments, nous retiendrons quant à nous, les deux suivants :

- Le rapport avec l'environnement, alors le système est ouvert.

- La conservation des systèmes, la survie.

$2^{\circ}$ Transition des configurations structurelles (Ivinza Lepapa, 2007)

Déjà Mintzberg (1979) introduisait la notion de transition dans configurations structurelles.

Parmi les quatre possibilités suggérées par Mintzberg pour exploiter les configurations structurelles, la transition des structures s'approche des finalités du modèle d'impact des TIC et donc de l'apparition des NFO. "La théorie des configurations structurelles peut aussi nous servir de base pour nous aider à comprendre comment et pourquoi les organisations entreprennent des transitions d'une structure à une autre» (p.415).

Il y a deux formes de transition, la première s'applique aux organisations qui commencent dans des environnements simples, la seconde s'applique aux organisations nées dans des environnements complexes.

Les études de transition ne peuvent être appréhendées que de façon diachronique (temporelle), car il s'agit des structures organiques, contrairement aux structures mécanistes qui peuvent être étudiées de façon synchronique (à un moment donné). «Les transitions structurelles suivent souvent avec retard les conditions qui les ont provoquées» (p.417).

Dans l'étude de l'impact organisationnel des technologies de l'information (de l'apparition des NFO), nous avions attiré l'attention sur les étapes du développement structurel : "à mesure que les organisations grandissent, elles passent par des périodes de transition structurelles, qui sont des changements de nature plutôt que des changements de degré» (p.223).

On retrouve aussi le concept de transition des configurations structurelles dans le «CBIS model» de Hossein Bidgoli (1997, p.5) qui cite « information system life cycle (ISLC) », comme l'un des sept éléments composant le CBIS. Selon lui, la vie d'un CBIS comprend quatre stades (introduction, croissance, maturité, déclin) qui dépendent des besoins des managers de chaque entreprise.

\section{Etat stationnaire et Homéostasie}

Les entreprises sont des systèmes ouverts d'apprentissage à décideurs multiples qui doivent assurer leur conservation et leur survie. Deux concepts permettent d'illustrer la survie des organisations :

- l'état stationnaire mieux utilisé en cybernétique

- et l'homéostasie découverte par le médecin français Claude Bernard.

Dans cet article, nous approcherons le changement organisationnel comme un phénomène homéostasique. 
Daniel DURAND (2006, p.19) relève les différences suivantes :

- «A la différence de la machine artificielle qui continue à exister sans fonctionner, l'arrêt de fonctionnement de l'organisme soumis à l'homéostasie signifie mort et corruption du corps ; le maintien de l'organisme vivant s'effectue en quelque sorte par un renouvellement constant de ses composants sans que son identité soit changée.

- Le système vivant fabrique lui-même ses propres constituants à partir d'éléments fournis par son environnement et réorganise sa propre structure ; la machine artificielle a été créée par un intervenant extérieur. »

L'homéostasie est un processus complexe et autonome de régulation et d'équilibration dans les systèmes vivants. Cannon le définit comme «l'ensemble des processus organiques qui agissent pour maintenir l'état stationnaire de l'organisme, dans sa morphologie et dans ses conditions intérieures, en dépit des perturbations extérieures.» (Durand, 2006, pp.18-19)

On peut donc, considérer l'homéostasie comme «la conjonction des processus par lesquels un système résiste au courant général de corruption et de dégénérescence. Elle désigne donc l'ensemble des rétroactions correctrices et régulatrices par lequel la dégradation déclenche la production et la réorganisation » (Boque, 1993, p.76).

Par le concept d'homéostasie, le changement organisationnel s'explique aussi aisément, suivant l'hypothèse d'Edgar Morin qui considère que la vie d'un système implique un double mouvement :1

- un mouvement de corruption et de désorganisation,

- un mouvement de fabrication et de réorganisation.

Grâce au concept systémique d'homéostasie nous pouvons dire que l'organisation évolue dans un environnement qui offre des opportunités d'une part et des menaces d'autre part. L'entreprise se comporte alors comme « un organisme vivant qui réagit aux changements et cherche à en tirer parti pour s'assurer un avantage sur ses concurrents. La combinaison de ces facteurs crée un environnement de plus en plus concurrentiel et changeant autour de l'entreprise, l'obligeant à des efforts d'innovation de plus en plus intenses. »(Jabes Jak, 1996, p.594).

\section{LA RESILIENCE STRATÉGIQUE}

La résilience stratégique est abordée en deux étapes. D'abord la compréhension du terme résilience et ensuite l'aspect stratégique qui en résulte.

\section{La resilience}

Le concept de résilience provient des travaux de recherche du docteur Boris Cyrulnik sur la capacité et le potentiel des enfants. En psychologie comme en management, la résilience représente la capacité et les attitudes d'un individu ou d'un système à surmonter l'incertitude. Il s'agit de vivre et de se développer en parvenant de vaincre les risques et à surmonter les chocs et traumatismes. Elle illustre la capacité adaptative de l'individu à mieux connaître ses limites et à vaincre ses peurs.

Nous basant sur cette compréhension, notre propos qui concerne la résilience des entreprises et des organisations, donc les NFO qui recherchent aujourd'hui l'économie d'adaptabilité. Cette adaptabilité doit permettre d'assurer la survie des entreprises. Cette survie marque le passage de l'ancienne organisation vers les NFO.

\section{La résilience stratégique}

En management on parlera de résilience stratégique qui n'est pas une manière de répondre à une crise passée, mais un modèle d'anticipation. Pour Garry Harmel et Liisa Valikangas, la résilience

\footnotetext{
${ }^{1}$ Cette partie résume Edgar Morin, 1977, pages 182 à 223 citée par BOQUE Jean-Michel, L'organisation en tant que système paradoxal, in BONAMI Michel et all, Management des systèmes complexes : «Pensée systémique et intervention dans les organisations », 1993, de Boeck page 15 .
} 
stratégique consiste à la capacité d'anticiper le changement avant que la nécessité de changer devienne une évidence.

Elle consiste à anticiper et à ajuster continuellement des tendances qui peuvent en permanence détériorer la capacité de l'activité corps de métier à générer des revenus.

La résilience stratégique ressemble à la veille stratégique, qui est basée sur le principe d'anticipation.

\section{RÉSILIENCE STRATÉGIQUE ET SYSTÈMES HOMÉOSTATIQUES UTILISANT LES TIC}

La résilience stratégique peut être opérée grâce à des modèles homéostasiques. Nous présenterons cidessous quelques modèles homéostasiques utilisant des TIC pour réaliser la résilience stratégique qui permettra la survie des entreprises et organisations et donc le passage de l'ancienne organisation vers les NFO.

Il existe plusieurs modèles dans la littérature du MIS, nous en avons choisi quelques-uns utilisés pour assurer la survie des organisations par une transition structurelle (diachronique) :

- MIT 90,

- Triangle stratégique,

- Holy Grail of IT,

- SAM-Strategic Alignment Model.

\section{Les travaux du MIT 90}

En 1984, à la Sloan Management School du MIT s'est constitué un groupe qui avait pour but d'étudier :

1. la turbulence de l'environnement (social, politique, technique et économique);

2. l'évolution des TI de la prochaine décennie suivant six aspects (matériels-hardware, logicielssoftware, réseaux, poste de travail, robotique, puces intelligentes).

Les organisations suivant le paradigme fonctionnaliste des écoles systémiques et de contingence structurelle doivent continuer à survivre devant la turbulence de l'environnement et de l'évolution rapide des TI. Ce qui implique, bien sûr, la maîtrise des changements qui se répercuteront sur les organisations.

$\underline{1^{\circ} \text { Les principales conclusions de la recherche (Scott Morton, 1991, pp.3-28) }}$

Les travaux du MIT 90 ont retenu six impacts majeurs des TI.

1- Les TI permettent d'apporter de profonds changements à la façon de travailler.

Trois sortes des travaux subissent le changement projeté : le travail de production, le travail de coordination, et le travail de management.

2- Les TI rendent possible l'intégration des fonctions à trois niveaux, à l'intérieur de l'organisation aussi bien avec d'autres organisations.

Cette intégration se manifeste sous quatre formes :

- à l'intérieur de la chaîne de valeur ajoutée,

- dans les liaisons de bout en bout de chaînes de valeur ajoutée entre organisations,

- dans la substitution de chaîne de valeur ajoutée, grâce à une sous-traitance ou une alliance,

- et dans les marchés électroniques.

3- Les TI créent des modifications dans le climat de concurrence de nombreuses branches professionnelles.

4- Les TI offrent de nouvelles opportunités stratégiques aux organisations qui révisent leurs missions et leur fonctionnement.

5- Pour réussir dans l'utilisation des TI, il faut apporter des changements dans le management et dans la structure de l'organisation. 
6- Un problème majeur des années 90 sera, pour les directions, de faire passer leurs organisations par toutes les transformations nécessaires pour réussir dans un environnement globalement concurrentiel.

$\underline{2^{\circ} \text { Les cinq forces de l'organisation du MIT } 90}$

Les impacts ci-dessus repérés correspondent à cinq forces de l'organisation qui sont : les technologies, les individus et rôles, les structures, le processus de management et la stratégie.

A vrai dire, le cadre du MIT 90, n'est que le raffinement du modèle de Leavitt (1968, p.325), auquel on a ajouté les environnements technologiques et socio-économiques externes.

Ces cinq forces sont représentées dans la figure ci-dessous (Scott Morton, 1991, p.35).

\section{Environnement Technologique Externe}

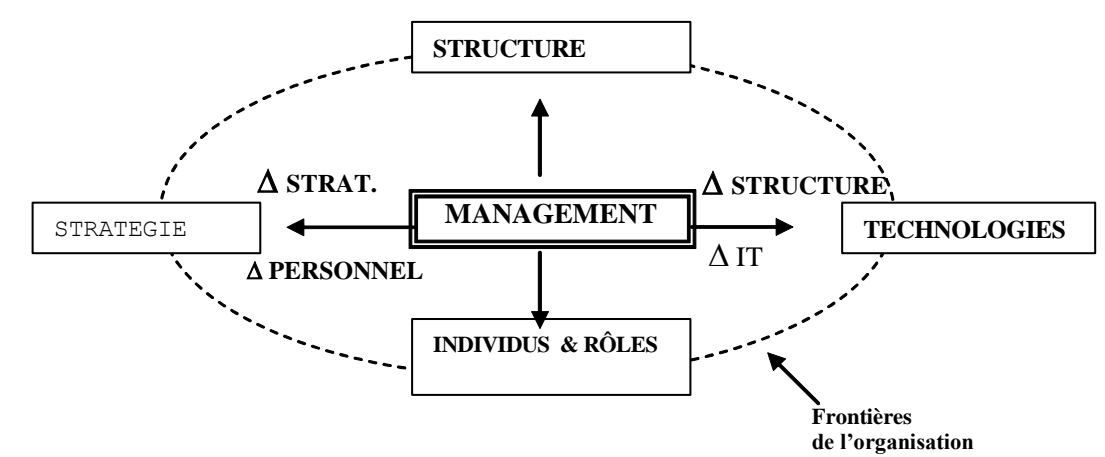

Environnement Socio- économique Externe

Figure 1. Le rôle du management dans le processus du changement (Cadre du MIT 90)

Le cadre du MIT90 propose cinq composants qui sont : la structure, la stratégie, les TI, les individus et le management. Ce modèle est du type homéostasique son utilisation assure la survie d'une organisation.

\section{Le triangle stratégique et l'organisation intelligente}

A la suite des travaux du MIT 90, Hubert Tardieu et Bernard Guthmann (1992) présentent le fondement du triangle stratégique, qui est un système d'auto - référence partielle comprenant la stratégie, la structure et les technologies de l'information.

"Le triangle stratégique ne s'est pas constitué en un jour. On peut identifier schématiquement trois grandes périodes :

- Les années 60, avec les travaux de R. Anthony et A. Chandler aux USA ainsi que J. Mélèse en France. L'entreprise est alors vue comme un système cybernétique.

- Les années 70, avec les travaux de H. Simon, M. Scott Morton et G. Davis aux USA ainsi que J.L. Le Moigne en France. L'entreprise est alors vue comme dotée d'une mémoire et capable d'organiser son processus de décision.

- Les années 80, avec les travaux de M. Porter, C. Wiseman et R. Wilenski aux USA. L'entreprise est désormais vue comme devant tirer partie d'opportunités stratégiques qui s'offrent à elles et donc concernée par la compréhension de son environnement." (Tardieu \& Guthmann, 1992, p.23)

Ce triangle est représenté à la figure 2 . 


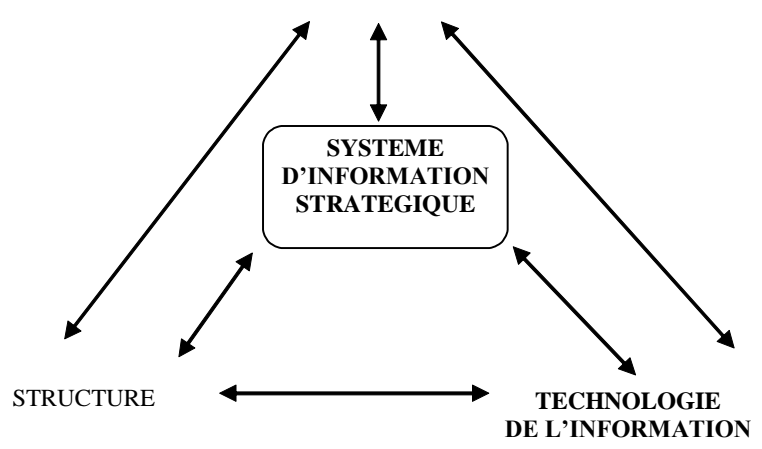

Figure 2. Le Triangle stratégique

Le triangle stratégique propose trois composants (TI, structure, stratégie) qui sont articulés autour du système d'information stratégique.

"La stratégie détermine les structures de l'entreprise, mais les technologies de l'information aident à déterminer la stratégie et à en faire le suivi. Les structures déterminent l'usage des technologies de l'information et permettent de déterminer les coups stratégiques qui eux-mêmes constituent la stratégie." (Tardieu \& Guthmann, 1992, p.67) Cette notion de système auto - référence était déjà évoquée dans l'article "Système d'information et pilotage de l'entreprise : quelques boucles étranges" (Tardieu \& Guthmann, 1992).

Le Grasce (Groupe de recherche sur l'adaptation, le systémique et la complexité économique) dirigé par le Professeur Jean- Louis Le Moigne, avait vers la même période inscrit dans son programme de recherche la théorie des organisations intelligentes en proposant (Bartoli \& Le Moigne, 1996) :

- des bases épistémologiques et paradigmatiques,

- des méthodologies de conception des systèmes d'information intelligents, supports de l'éco pilotage stratégique,

- et quelques exemples des organisations intelligentes.

Ce modèle est aussi du type homéostasique, son opérationnalisation peut assurer la survie d'une organisation dans la perspective de la résilience stratégique.

\section{The Holy Grail of IT: a circle of forces}

Bob Galliers et Walter Baets (1998, pp.3-6) reprenant Baskerville et Smithson (1995) considèrent que très peu des études et recherches concernant les impacts organisationnels des TI sont descriptives et holistiques. Cela proviendrait de cinq raisons majeures (notre traduction) :

1. Les professionnels des TI tendent toujours à généraliser une expérience heureuse en paradigme managérial de succès.

2. Les chercheurs des TI cherchent une formule universelle qui va transformer n'importe quel type d'organisation d'une situation médiocre en une situation excellente.

3. Les chercheurs des TI donne une préséance aux TI et néglige d'autres facteurs important que sont le social et l'environnement.

4. Les théoriciens du management semblent incapables à lier l'imprévisibilité de la nature humaine dans l'organisation et son contexte culturel.

5. Plusieurs facteurs critiques influencent les impacts organisationnels des TI et les formes émergentes des organisations (organisation intelligente). 


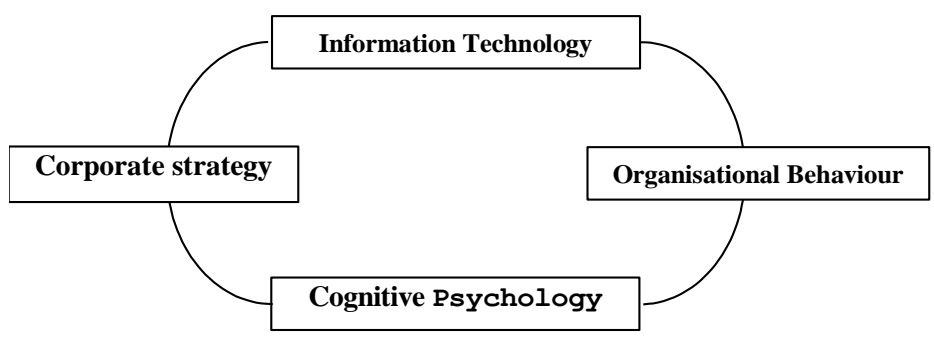

Figure 3. Circle of forces for the consideration of IT and organizational transformation

Galliers et Baets (1998, pp.6-8) considèrent que quatre forces expliquent les impacts organisationnels des TI, suivant la figure 3 ci-dessus.

Information technology (technologie de l'information). Ces quatre dernières décennies, les technologies de l'information ont marqué les différents changements organisationnels.

Corporate strategy (Stratégie d'entreprise). Deux tendances marquent la stratégie de l'entreprise avec les TI. La première tendance concernant l'insertion des TI dans la stratégie de l'entreprise, la seconde tendance concernant le "design school" est influencée surtout par la pensée systémique et contingencialiste.

Organizational behaviour (Comportement organisationnel). Cette force est remarquable pour les organisations intelligentes, qui permettent aux membres d'évoluer dans le même ordre que l'entreprise.

Cognitive Psychology (Psychologie cognitive). Cette tendance devient pertinente avec l'émergence des organisations intelligentes et des réseaux neuronaux. C'est ce que le programme du GRASCE appelle intelligence organisationnelle (Bartoli et Le Moigne, 1996).

Ce modèle est aussi du type homéostasique, son opérationnalisation est difficile, mais il peut assurer la survie d'une organisation dans la perspective de la résilience stratégique.

\section{L'alignement stratégique des TI comme modèle de changement organisationnel}

$1^{\circ}$ Cadre théorique. (Venkatraman, 1991,1995; Henderson et Venkatraman, 1994, Henderson, Venkatraman and Oldach, 1996)

Dans une de ses conclusions, le rapport du MIT90 a montré que dans l'environnement concurrentiel actuel, aucune entreprise ne peut se passer des TI. Pour fonctionner dans cet environnement, les entreprises doivent aligner consciemment leurs stratégies et leurs structures sur les TI. Cet alignement doit être dynamique, c'est à dire évolutif entre le contexte stratégique de l'entreprise et des TI dans le cadre d'un modèle appelé SAM (Strategic Alignement Model).

Le modèle théorique d'alignement stratégique (SAM) proposé par Venkatraman (1991, pp.122-158) comprend quatre composants : la stratégie commerciale (stratégie d'entreprise), l'infrastructure et les processus de l'entreprise (infrastructure organisationnelle), la stratégie des TI, l'infrastructure et les processus des TI (infrastructure des SI).

Ce modèle est analysé suivant trois types de relation :

- bivariée verticale qui représente l'intégration stratégique (stratégie d'entrepriseinfrastructure organisationnelle, stratégie des TI- infrastructure des TI),

- bivariée horizontale qui représente l'intégration fonctionnelle (stratégie d'entreprisestratégie des TI, infrastructure organisationnelle- infrastructure des TI),

- multivariée qui donne les quatre triades du schéma d'alignement stratégique : potentiel concurrentiel (stratégie d'entreprise- infrastructure des SI - infrastructure organisationnelle), potentiel technologique (stratégie d'entreprise- stratégie des TI infrastructure des SI) valeur de l'entreprise (stratégie d'entreprise- stratégie des TI infrastructure organisationnelle), niveau de service (stratégie d'entreprise- stratégie des TI -infrastructure des SI). 


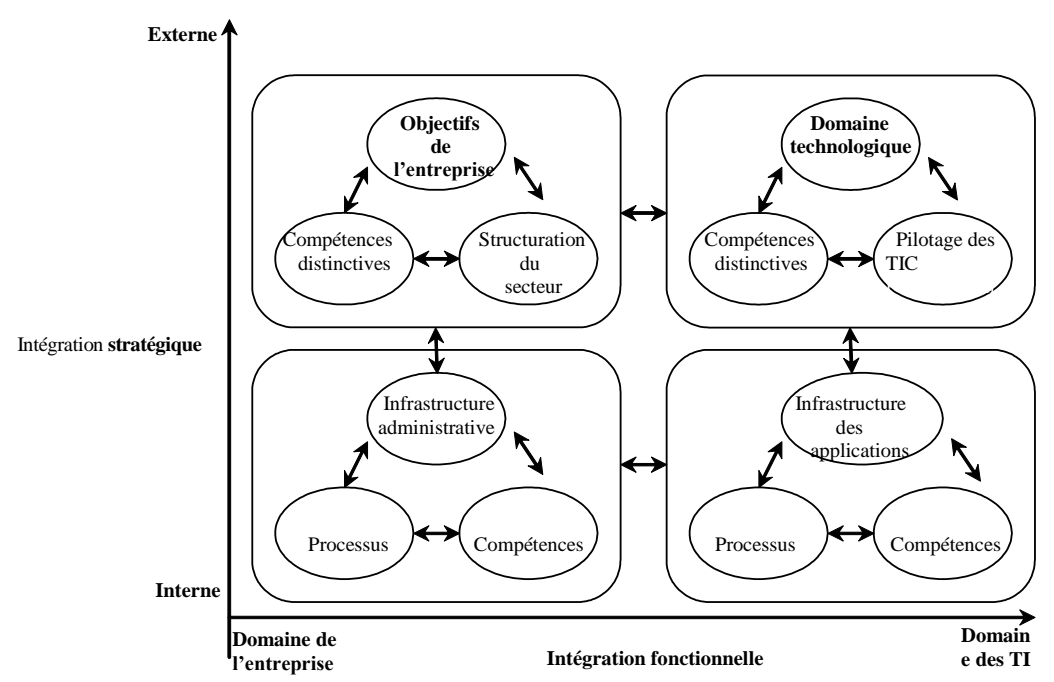

Figure 4. Le modèle d'alignement stratégique

$\underline{2^{\circ} \text { Les niveaux de reconfiguration d'entreprises provoquées par les technologies de l'information }}{ }^{2}$.

Ce modèle propose cinq niveaux de reconfiguration provoqués par les TI : (1) Exploitation en local, (2) Intégration interne, (3) Reconception des processus de gestion, (4) Reconception du réseau de gestion, (5) Redéfinition des objectifs de l'entreprise.

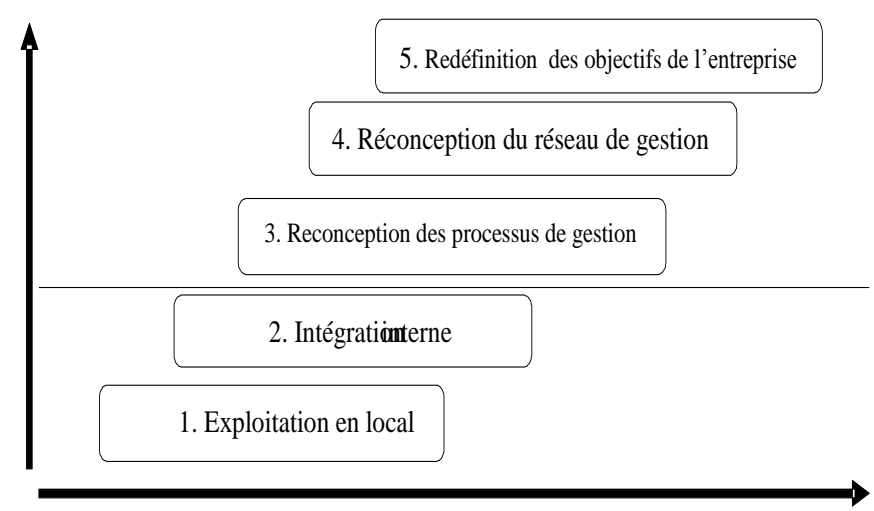

Figure 5. Les cinq niveaux de reconfiguration provoqués par les technologies de l'information

\section{Le niveau 1 : Exploitation en local.}

Il concerne l'utilisation des technologies de l'information dans le cadre d'une fonction de l'entreprise telle que la production ou le commercial ce qui implique la mise en œuvre d'applications des technologies de l'information destinées améliorer l'efficacité des opérations.

2- Le niveau 2 : Intégration interne.

C'est une extension logique du niveau précédent en ce sens que les possibilités des technologies de l'information sont exploitées dans toutes les fonctions de l'entreprise où cela est faisable.

\footnotetext{
${ }^{2}$ Ce paragraphe s'inspire complètement de Venkatraman, 1991, pp.122-158.
} 
Deux types d'intégration sont possibles :

- l'intégration technique : c'est l'intégration des différents systèmes d'applications utilisant une plate-forme informatique commune,

- l'intégration organisationnelle des différents rôles et responsabilités exploitant les possibilités d'intégrations techniques.

En d'autres termes, la mise en œuvre d'une plate-forme informatique commune sert à intégrer les activités de gestion de l'organisation ; ce qui doit en améliorer potentiellement l'efficacité.

3- Le niveau 3 : Reconception des processus de gestion.

Elle concerne la reconfiguration de la gestion en utilisant les technologies de l'informatique comme levier central. Au lieu de traiter les processus de gestion existants comme une contrainte dans la définition d'une infrastructure informatique optimale, c'est le processus de gestion lui-même qui est réétudié pour exploiter au mieux les possibilités offertes par les technologies de l'information. Ce qui est un reflet d'efforts conscients de création d'une homogénéité.

\section{4- Le niveau 4 : Réconception du réseau de gestion}

Elle consiste à se servir des technologies de l'information pour mettre en place un système d'information qui va utiliser les fournisseurs, clients et autres tiers capables de contribuer à l'objectif de la firme. En d'autres termes, il s'agit de quitter l'organisation traditionnelle pour aller vers une organisation «virtuelle » ou en «réseau » qui permettra d'atteindre un objectif particulier. L'intégration électronique des partenaires externes devient ainsi le problème dominant du management stratégique.

\section{5 - Le niveau 5 : Redéfinition des objectifs de l'entreprise}

A ce niveau, l'organisation décide de rompre avec le passé et décide d'exploiter les nouvelles technologies en termes de marchés ou de produits.

\section{6- Conclusion}

Les niveaux 1 et 2 sont évolutifs, les niveaux 3 à 5 sont révolutionnaires. Les deux premiers niveaux ont un caractère évolutif et se contentent de modifications relativement incrémentales des processus existants.

En revanche, les trois autres niveaux sont conceptualisés comme quelque chose de révolutionnaire et ils exigent des changements de fond dans la nature même du processus de gestion.

Ce modèle est aussi du type homéostasique. C'est le modèle plus utilisé dans le MIS pour assurer la survie d'une organisation dans la perspective de la résilience stratégique.

\section{CONCLUSION GÉNÉRALE}

Dans la première section on a présenté succinctement les origines et les catalyseurs des NFO et avons rappelé aussi notre conception des entreprises et des organisations du point de vue systémique.

Pour la bonne compréhension de notre approche, nous avons apporté quelques éclaircissements sur les expressions changement organisationnel et transition structurelle. A la fin de cette section, nous avons contrasté les concepts état stationnaire et l'homéostasie du point de vue organisationnel.

La résilience stratégique a été abordée en deux étapes. D'abord la compréhension du terme résilience et ensuite l'aspect stratégique qui en résulte.

La résilience stratégique peut être opérée grâce à des modèles homéostasiques. Nous avons présenté quelques modèles homéostasiques utilisant des TIC, pour réaliser la résilience stratégique qui permettra la survie des entreprises et organisations et donc le passage de l'ancienne organisation vers les NFO. 


\section{RÉFÉRENCES}

BARTOLI, Jacques André \& LE MOIGNE, Jean Louis (1996). Organisation intelligente et système d'information stratégique. Economica gestion, Paris.

BIDGOLI, Hossein (1997). Modern Information Systems for Managers. Academic Press, California. BOQUE, Jean-Michel (1993). "L'organisation en tant que système paradoxal". BONAMI, Michel et al. (1993). Management des systèmes complexes: Pensée systémique et intervention dans les organisations. de Boeck.

DURAND, Daniel (2006). La Systémique. Presses Universitaires de France (PUF), Paris.

GALLIERS, Robert D. \& BAETS, Walter R. (1998). Information Technology and organizational transformation : Innovation for the $21^{\text {st }}$ Century organisation. John Wiley and Sons, New York.

GODELIER, Eric (1998). "Le changement dans les entreprises : crise ou mutation". Revue Française de Gestion, $\mathrm{n}^{\circ} 120$, Septembre - Octobre 1998, page 26 et sq.

IVINZA LEPAPA, Alphonse (2019). "NFO : Nouvelles Formes d'Organisation; entreprises réseau, virtuelle, numérique et Télétravail". Bookelis, Paris.

IVINZA LEPAPA, Alphonse (2016). Informatique Stratégique : Cadre théorique et Applications dans les entreprises congolaises. Bookelis, Paris.

IVINZA LEPAPA, Alphonse (2010). Analyse de l'introduction de l'EDI dans les entreprises congolaises : une contribution à l'impact organisationnel des TI. Tome 1, EUE.

IVINZA LEPAPA, Alphonse (2007). Analyse de l'introduction de l'EDI dans les entreprises congolaises : une contribution à l'impact organisationnel des TI. Tome 2, ULB, Solvay Business School.

MINTZBERG, Henry (1979). Structure et dynamique des organisations.

SEGUIN, F. \& CHANLAT, J.F. (1983). L'analyse des organisations, une anthologie sociologique, Tome I Les théories de l'organisation. Gaëtan Morin éditeur, Paris.

SCOTT MORTON, Michael S. (ed.) (1991). The Corporation of the 1990's, Information technology and Organization transformation. New York, Oxford University Press; pour l'édition française L'entreprise compétitive au futur: Technologies de l'information et transformation de l'organisation. Les éditions d'organisations, Paris, éd.1995.

TARDIEU, Hubert \& GUTHMANN, Bernard (1992). Le Triangle Stratégique : Stratégie, Structure et Technologie de l'information. Edition de l'organisation, Paris.

TARDIEU, Hubert \& THEYS, Michel (1987). "Systèmes d'information et pilotage de l'entreprise : quelques boucles étranges". Revue Internationale du systémique, numéro spécial "L'entreprise système", Vol.1, nº 4 .

VENKATRAMAN, N. (1991). IT-Induced Business Reconfiguration. M.S. Scott Morton, The Corporation of the 1990's, pp.122-158.

WILKIN, Luc (1986). Informatique et Organisations. Actes du colloque de Nivelles : L'ordinateur, L'homme et L'organisation $2^{\mathrm{e}}$ partie, PUB.

WILKIN, Luc (1999). Principes généraux d'organisation et de gestion. 2e édition, PUB. 
